\title{
Results on $b$ hadron properties in CMS
}

\author{
Thomas Madlener $^{1, \star}$ on behalf of the CMS collaboration \\ ${ }^{1}$ Institute of High Energy Physics (HEPHY), Vienna
}

\begin{abstract}
The lifetime is among the fundamental properties of particles and is one of the important observables in heavy hadrons allowing to test the theoretical tools describing their physics. This report presents lifetime measurements of $\mathrm{B}^{0}, \mathrm{~B}_{s}^{0}, \Lambda_{b}^{0}$ and $\mathrm{B}_{c}^{+}$hadrons performed on data, corresponding to $19.7 \mathrm{fb}^{-1}$, collected by the CMS experiment at the LHC at a center-of-mass energy of $\sqrt{s}=8 \mathrm{TeV}$. The $\mathrm{B}^{0}$ lifetime is measured to be $453.0 \pm$ 1.6 (stat) \pm 1.5 (syst) $\mu \mathrm{m}$ in $\mathrm{J} / \psi \mathrm{K}_{S}^{0}$ and $457.8 \pm 2.7$ (stat) \pm 2.7 (syst) $\mu \mathrm{m}$ in $\mathrm{J} / \psi \mathrm{K}^{*}(892)^{0}$. The $\mathrm{B}_{s}^{0}$ meson lifetime is measured in two decay modes with differing contributions from the heavy and light eigenstates, resulting in two different measured lifetimes: $504.3 \pm$ 10.5 (stat) \pm 3.7 (syst) $\mu \mathrm{m}$ in $\mathrm{J} / \psi \pi^{+} \pi^{-}$and $443.9 \pm 2.0$ (stat) \pm 1.2 (syst) $\mu \mathrm{m}$ in $\mathrm{J} / \psi \phi(1020)$ respectively. The lifetime of the $\Lambda_{b}^{0}$ hadron is found to be $443.1 \pm 8.2$ (stat) \pm 2.7 (syst) $\mu \mathrm{m}$. The $\mathrm{B}_{c}^{+}$lifetime is measured with respect to the $\mathrm{B}^{+}$lifetime and is found to be $162.3 \pm$ 8.2 (stat) \pm 4.7 (syst) $\pm 0.1\left(\tau_{\mathrm{B}^{+}}\right) \mu \mathrm{m}$.
\end{abstract}

\section{Introduction}

Precise lifetime measurements of heavy hadrons play an important role in the study of nonperturbative Quantum Chromodynamics (QCD). The QCD inspired Heavy Quark Expansion (HQE) model describes the weak decays of hadrons containing a heavy quark and provides accurate estimates of the ratio of their lifetimes [1]. The large production rate of $b$ hadrons at the LHC and the detection capabilities of the CMS detector allow to make precise $b$ hadron lifetime measurements, which in turn make it possible to test these predictions.

In this report the measurement of the $\mathrm{B}^{0}, \mathrm{~B}_{s}^{0}, \mathrm{~B}_{c}^{+}$and $\Lambda_{b}^{0}$ hadrons lifetimes at the center-of-mass energy of $\sqrt{s}=8 \mathrm{TeV}$ by the CMS collaboration [2] are discussed.

The lifetime measurement is based on the reconstruction of the transverse decay length $L_{x y}$, where $\vec{L}_{x y}$ is the flight distance vector from the primary vertex to the decay vertex of the $b$ hadron, projected onto the transverse momentum $\vec{p}_{T}$ of the $b$ hadron momentum. The proper decay time times the speed of light $c$ is measured via

$$
c t=c L_{x y} \frac{M}{p_{T}},
$$

where $M$ is the world-average value of the b hadron mass [3].

\footnotetext{
^e-mail: thomas.madlener@cern.ch
} 


\section{Dataset and event selection}

The data used in the analysis has been been collected by the CMS experiment at the LHC in 2012 in proton-proton collisions at a center-of-mass energy of $\sqrt{s}=8 \mathrm{TeV}$ and corresponds to an integrated luminosity of $19.7 \mathrm{fb}^{-1}$.

The analysis is based on a data sample collected with a trigger requiring two oppositely charged muons within the pseudorapidity region $|\eta|<2.2$. The dimuon is requested to have a transverse momentum, $p_{T}$, greater than $7.9 \mathrm{GeV}$ and to have an invariant mass $2.8<M\left(\mu^{+} \mu^{-}\right)<3.35 \mathrm{GeV}$. Both muons must have a distance of closest approach to the event vertex in the transverse plane smaller than $0.5 \mathrm{~cm}$. A fit to a common vertex must have a $\chi^{2}$ probability greater than $0.5 \%$. The offline selection criteria of the dimuon system replicate the trigger selection or impose slightly stricter requirements, such as the invariant mass being within $150 \mathrm{MeV}$ of the world-average value [3] unless stated otherwise.

Muons are identified using the standard CMS muon reconstruction procedure [4], which requires multiple hits in the detector with a consistent trajectory. Tracks are required to fulfill the standard CMS high-purity requirements [5] and have $p_{T}$ greater than $0.5 \mathrm{GeV}$.

\section{Reconstruction of $b$ hadrons}

The offline selection starts by reconstructing $\mathrm{J} / \psi$ candidates from pairs of oppositely charged muons. Candidate $b$ hadrons are built by combining a $\mathrm{J} / \psi$ candidate with reconstructed neutral particles or track(s) depending on the decay mode under consideration. Intermediate neutral particles are reconstructed by combining pairs of oppositely charged tracks with appropriately assigned masses. A candidate $b$ hadron is fitted to a common vertex constraining the dimuon mass to the world-average $\mathrm{J} / \psi$ mass [3]. In fits including a $\mathrm{K}_{S}^{0}$ or $\Lambda^{0}$ hadron the world-average mass values are used for these particles. Primary Vertices (PV) are fitted from the reconstructed tracks in the event using the beamspot - the estimated proton-proton interaction region - as a constraint. The PV with the smallest pointing angle is used, where the pointing angle is defined as the angle between the reconstructed $b$ hadron momentum and the vector joining the PV and the decay vertex.

\subsection{Reconstruction of $\mathrm{B}^{0}, \mathrm{~B}_{s}^{0}$ and $\Lambda_{b}^{0}$ hadrons}

The $\mathrm{B}^{0}, \mathrm{~B}_{s}^{0}$ and $\Lambda_{b}^{0}$ hadrons are reconstructed in the following decay channels:

$$
\begin{array}{ll}
\mathrm{B}^{0} \rightarrow \mathrm{J} / \psi \mathrm{K}_{S}^{0} & \rightarrow \mu^{+} \mu^{-} \pi^{+} \pi^{-} \\
\mathrm{B}^{0} \rightarrow \mathrm{J} / \psi \mathrm{K}^{*}(892)^{0} & \rightarrow \mu^{+} \mu^{-} \mathrm{K}^{ \pm} \pi^{\mp} \\
\mathrm{B}_{s}^{0} \rightarrow \mathrm{J} / \psi \phi(1020) & \rightarrow \mu^{+} \mu^{-} \mathrm{K}^{+} \mathrm{K}^{-} \\
\mathrm{B}_{s}^{0} \rightarrow \mathrm{J} / \psi \pi^{+} \pi^{-} & \rightarrow \mu^{+} \mu^{-} \pi^{+} \pi^{-} \\
\Lambda_{b}^{0} \rightarrow \mathrm{J} / \psi \Lambda^{0} & \rightarrow \mu^{+} \mu^{-} p \pi^{-}
\end{array}
$$

The $\mathrm{B}^{0}, \mathrm{~B}_{s}^{0}$ and $\Lambda_{b}^{0}$ hadrons must have $p_{T}>13 \mathrm{GeV}$, except for the $\mathrm{B}_{s}^{0} \rightarrow \mathrm{J} / \psi \phi(1020)$ channel, where no such requirement is imposed. The vertex fit $\chi^{2}$ probability has to be greater than $0.1 \%$ for the latter decay only. For all channels the lifetime measurement is restricted to events for which the $c t$ of the $b$ hadron is larger than $0.02 \mathrm{~cm}$ to avoid reconstruction and resolution effects in the low- $c t$ region. 


\subsection{Reconstruction of $\mathbf{B}_{c}^{+} \rightarrow \mathbf{J} / \psi \pi^{+}$and $\mathbf{B}^{+} \rightarrow \mathbf{J} / \psi \mathbf{K}^{+}$}

Both decays are reconstructed using the same algorithm and selection criteria and as a result the dependence of the efficiencies on the proper decay time is similar.

The dimuon invariant mass must lie in the range $\pm 3 \sigma$ from the nominal $\mathrm{J} / \psi$ mass, where $\sigma$ is the average resolution for the $\mathrm{J} / \psi$ signal depending on the $\mathrm{J} / \psi$ pseudorapidity. The vertex $\chi^{2}$ probability for the dimuon has to be greater than $1 \%$ and the $b$ hadron vertex $\chi^{2}$ probability has to greater then $5 \%$. The $p_{T}$ of the $b$ hadron and the charged track have to be greater than $10 \mathrm{GeV}$ and 3.3 $\mathrm{GeV}$, respectively. The lifetime measurement is limited to $c t>0.01 \mathrm{~cm}$ to ensure a constant ratio of the meson efficiencies. Additionally, the angle $\cos \theta$ is required to be greater than 0.98 , where $\cos \theta=\vec{L}_{x y} \cdot \vec{p}_{T, B} /\left(\left|\vec{L}_{x y}\right| \cdot\left|\vec{p}_{T, B}\right|\right)$ and $\vec{L}_{x y}$ and $\vec{p}_{T, B}$ refer to the transverse decay length and transverse momentum of the $\mathrm{B}^{+}$and $\mathrm{B}_{c}^{+}$mesons.

\section{$4 \mathrm{~B}^{0}, \mathbf{B}_{s}^{0}$ and $\Lambda_{b}^{0}$ lifetime measurements}

The neutral $\mathrm{B}_{q}^{0}$ decay rate is characterized by the average decay width, $\Gamma_{q}=\left(\Gamma_{L}^{q}+\Gamma_{H}^{q}\right) / 2$, and the decay width difference, $\Delta \Gamma_{q}=\Gamma_{L}^{q}-\Gamma_{H}^{q}$, where $\Gamma_{L, H}^{q}$ are the decay widths of the light (L) and heavy $(\mathrm{H})$ mass eigenstates. The $\mathrm{B}^{0}$ system has a small lifetime difference compared to the average lifetime $\frac{\Delta \Gamma_{d}}{\Gamma_{d}}=(-0.3 \pm 1.5) \%$ [3], and thus the the $c t$ distribution can be treated as an exponential. However, in the $\mathrm{B}_{s}^{0}$ system, $\frac{\Delta \Gamma_{s}}{\Gamma_{s}}=(12.4 \pm 1.1) \%$ [3] and the deviation from an exponential has to be considered for the measurement.

Two lifetimes associated to the $\mathrm{B}_{s}^{0}$ are measured in the decay channels $\mathrm{J} / \psi \pi^{+} \pi^{-}$and $\mathrm{J} / \psi \phi(1020)$. The latter final state is an admixture of two CP-even and one CP-odd state and can be used to measure the effective lifetime of the $\mathrm{B}_{s}^{0}$ system. This measurement is complementary to the weak mixing phase analysis in the same channel [6]. The $\mathrm{J} / \psi \pi^{+} \pi^{-}$final state is reconstructed in the mass window $0.9240<M\left(\pi^{+} \pi^{-}\right)<1.204 \mathrm{GeV}$, dominated by the $f_{0}(980)$ resonance [7, 8]. Thus, this is a CP-odd final state and the measured lifetime is related to the heavy $\mathrm{B}_{s}^{0}$ mass eigenstate, $\tau_{\mathrm{B}_{s}^{0}}^{\mathrm{CP} \text {-odd }} \approx 1 / \Gamma_{H}$.

\subsection{Data modelling}

For all three hadrons a three-dimensional unbinned maximum-likelihood fit using the $b$ hadron mass, $c t$ and the per-event $c t$ uncertainty, $\sigma_{c t}$, is performed. For the $\mathrm{B}_{s}^{0}$ modes an extended unbinned maximum-likelihood fit is used.

The invariant mass distribution for the signal is modelled with a sum of two Gaussian functions, except for the $\mathrm{B}_{s}^{0}$, where one Gaussian is sufficient for describing the data. The combinatorial background is modelled by a linear polynomial or an exponential in the case of the $\mathrm{B}_{s}^{0} \rightarrow \mathrm{J} / \psi \phi(1020)$. For the $\mathrm{B}_{s}^{0} \rightarrow \mathrm{J} / \psi \pi^{+} \pi^{-}$decay additional terms are added for describing backgrounds from partially reconstructed or misidentified $b$ hadron decays: The decays $\mathrm{B}^{0} \rightarrow \mathrm{J} / \psi \pi^{+} \pi^{-}$and $\mathrm{B}_{(s)}^{0} \rightarrow \mathrm{J} / \psi \mathrm{h}_{1}^{+} \mathrm{h}_{2}^{-}$, where $\mathrm{h}_{1}^{+}$and $\mathrm{h}_{2}^{-}$are charged hadron tracks that are not both pions, are modelled by two separate Gaussian functions while the shape of the $\mathrm{B}^{+} \rightarrow \mathrm{J} / \psi \mathrm{K}^{+}$are taken from simulations.

The $c t$ signal distribution is modelled by an exponential function convolved with the detector resolution and then multiplied by an efficiency function. The backgrounds are described by a superposition of exponential functions likewise convolved with the resolution. The resolution is described by a Gaussian function with the event-per-event width taken from the $c t$ uncertainty distribution and are in the order of some $\mu \mathrm{m}$.

The signal and background $\sigma_{c t}$ distributions are modelled with a Gaussian function convolved with either two exponential functions or the sum of two gamma functions. 
The invariant mass and $c t$ distributions obtained from data together with the superimposed fit results are shown in Figs. 1-3
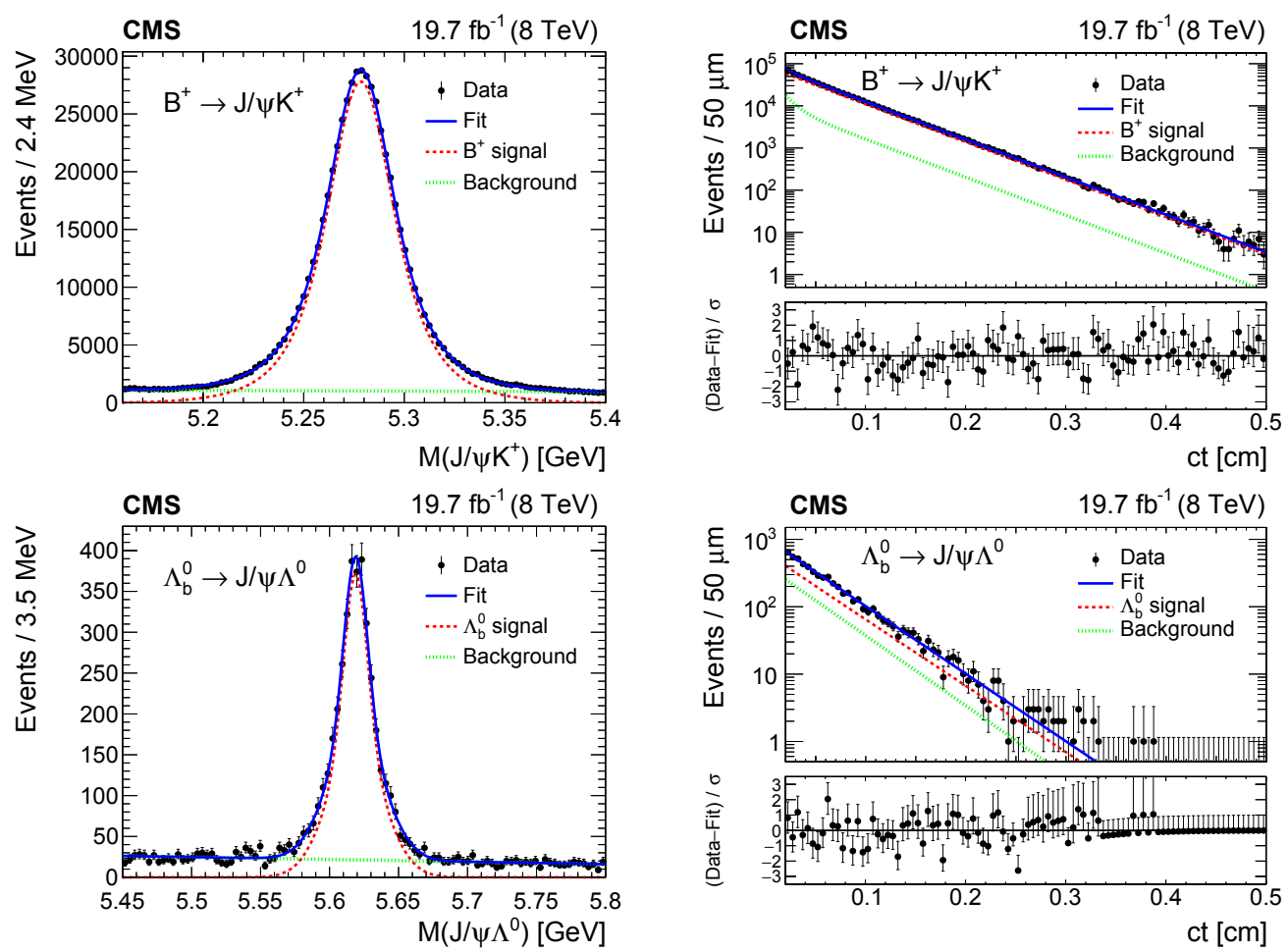

Figure 1. Invariant mass (left) and ct distributions (right) for $\mathrm{B}^{+}$(top) and $\Lambda_{b}^{0}$ candidates (bottom) together with projections of the fit to the data. The contributions from signal (dashed), background (dotted) and the sum of the two (solid) are shown. The bottom panels of the right figures show the differences between the observed data and the fit divided by the data uncertainty [2].

\subsection{Efficiency}

To determine the reconstruction and selection efficiency as a function of $c t$, fully simulated MC samples are used for each decay mode. The efficiency is defined as the generated $c t$ distribution of the selected events after reconstruction divided by the $c t$ distribution obtained from an exponential decay with the lifetime set to the value used in the generation of the events. For the $\mathrm{B}_{s}^{0} \rightarrow \mathrm{J} / \psi \phi(1020)$ channel the sum of the two exponentials generated with the theoretical $\mathrm{B}_{s}^{0} \rightarrow \mathrm{J} / \psi \phi(1020)$ decay rate model [9] is used as the denominator. The efficiencies for the different decay modes are shown in Fig. 4.

\section{$5 \mathrm{~B}_{c}^{+}$lifetime measurements}

In the current $\mathrm{B}_{c}^{+}$decay model different processes contribute to the decay width. The weak decay can either occur through the $b$ or $c$ quark decaying with the other quark as spectator or through an annihilation process, which is predicted to contribute up to $10 \%$ of the decay width [10]. Thus lifetime 

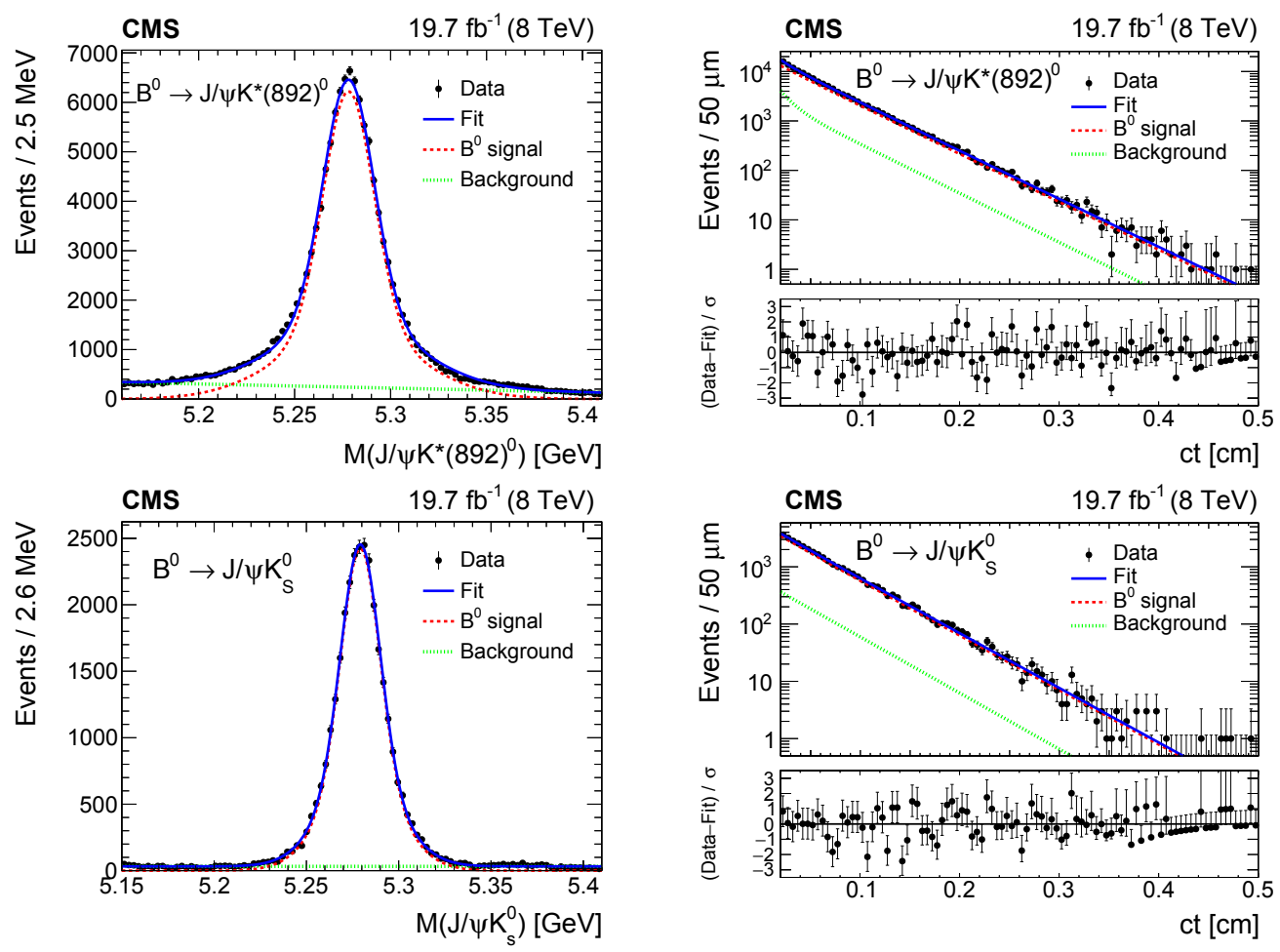

Figure 2. Invariant mass (left) and $c t$ distributions (right) for $\mathrm{B}^{0}$ candidates reconstructed from $\mathrm{J} / \psi \mathrm{K}^{*}(892)^{0}$ decays (top) and $\mathrm{J} / \psi \mathrm{K}_{S}^{0}$ decays (right) together with projections of the fit to the data. The contributions from signal (dashed), background (dotted) and the sum of the two (solid) are shown. The bottom panels of the right figures show the differences between the observed data and the fit divided by the data uncertainty [2].

measurements can be used to test the $\mathrm{B}_{c}^{+}$decay model. Additionally, recent lifetime measurements reported by the LHCb collaboration $[11,12]$ are significantly larger than those measured at the Tevatron [13-15]. A measurement by CMS may help to resolve this ambiguity in experimental results.

The $\mathrm{B}_{c}^{+}$lifetime measurement uses the method developed by the LHCb collaboration $[11,12]$, which exploits the precise knowledge of the $\mathrm{B}^{+}$lifetime [3] to obtain the $\mathrm{B}_{c}^{+}$lifetime by measuring the difference in total width between the $\mathrm{B}_{c}^{+}$and the $\mathrm{B}^{+}$,

$$
\Delta \Gamma \equiv \Gamma_{\mathrm{B}_{c}^{+}}-\Gamma_{\mathrm{B}^{+}}=\frac{1}{\tau_{\mathrm{B}_{c}^{+}}}-\frac{1}{\tau_{\mathrm{B}^{+}}}
$$

The signal decay time distribution $N_{\mathrm{B}}(c t)$ can be expressed as the product of an exponential decay function $E\left(c t \mid \tau_{\mathrm{B}}\right)=\exp \left(-c t / \tau_{\mathrm{B}}\right)$ convolved with the time resolution function of the detector $R(c t)$ and the efficiency function $\epsilon_{\mathrm{B}}(c t)$. The ratio of $\mathrm{B}_{c}^{+}$to $\mathrm{B}^{+}$events at any given proper time then becomes

$$
\frac{N_{\mathrm{B}_{c}^{+}}(c t)}{N_{\mathrm{B}^{+}}(c t)}=\mathcal{R}(c t)=\frac{\left[E\left(c t \mid \tau_{\mathrm{B}_{c}^{+}}\right) \otimes R(c t)\right] \epsilon_{\mathrm{B}_{c}^{+}}(c t)}{\left[E\left(c t \mid \tau_{\mathrm{B}^{+}}\right) \otimes R(c t)\right] \epsilon_{\mathrm{B}^{+}}(c t)} .
$$



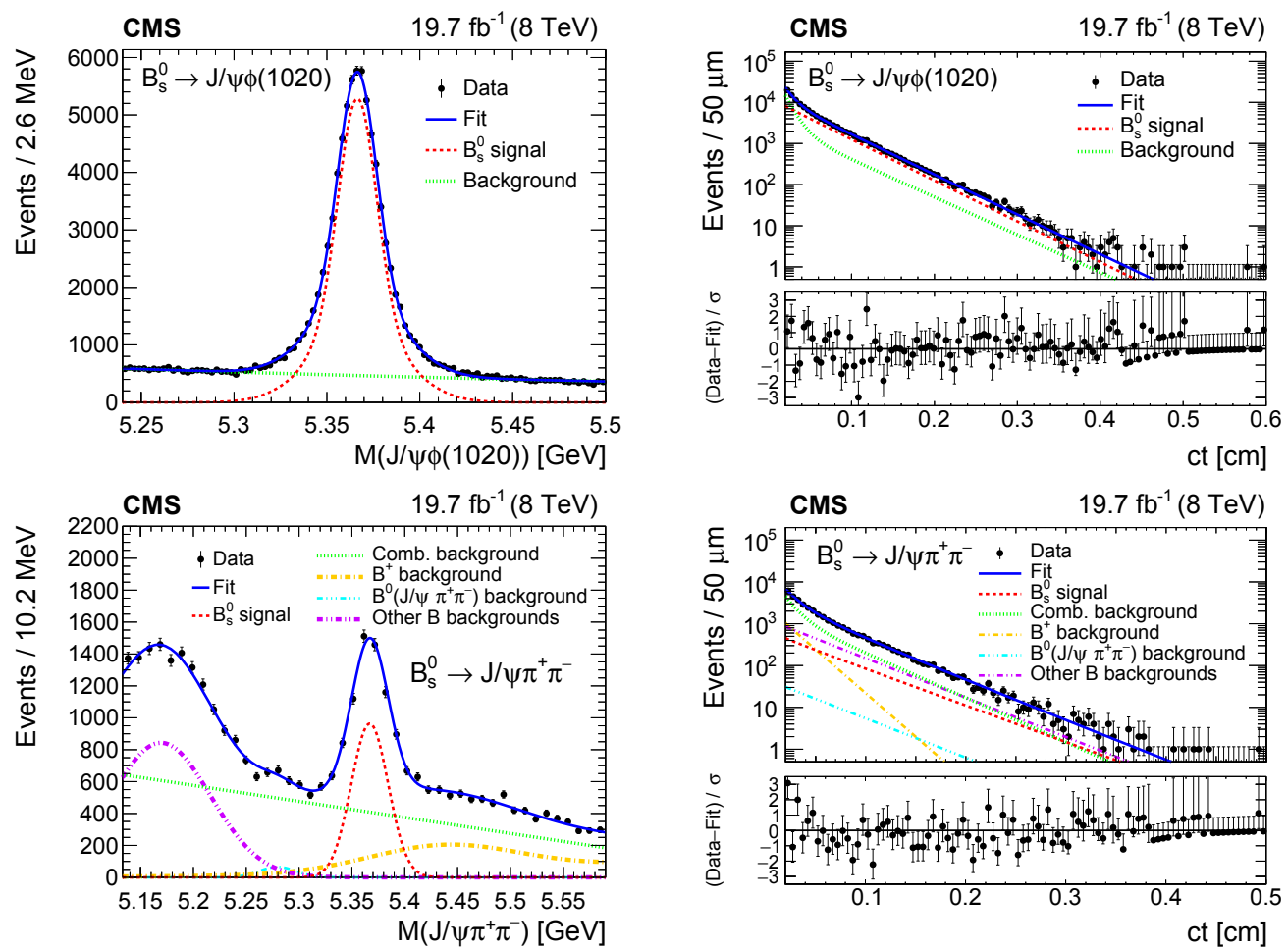

Figure 3. Invariant mass (left) and $c t$ distributions (right) for $\mathrm{B}_{s}^{0}$ candidates reconstructed from $\mathrm{J} / \psi \phi(1020)$ decays (top) and from $\mathrm{J} / \psi \pi^{+} \pi^{-}$decays (bottom) together with projections of the fit to the data. The full fit function (solid) and the signal (dashed) are shown for both decays. The total background (dotted) is shown for the top plots. For the bottom plots the combinatorial background (dotted), misidentified $\mathrm{B}^{+} \rightarrow \mathrm{J} / \psi \mathrm{K}^{+}$(dash-dotted), $\mathrm{B}^{0} \rightarrow$ $\mathrm{J} / \psi \pi^{+} \pi^{-}$(dashed-dotted-dotted-dotted), and partially reconstructed and misidentified B contributions (dasheddotted-dotted) are shown separately. The bottom panels of the right figures show the differences between the observed data and the fit divided by the data uncertainty [2].

Studies using simulated pseudo events have been used to verify that Eq. 3 is not significantly affected by the time resolution and thus it can be simplified to

$$
\mathcal{R}(c t)=R_{\epsilon}(c t) \exp (-\Delta \Gamma t)
$$

where residual effects of the time resolution are absorbed into the ratio of the efficiency functions $R_{\epsilon}(c t)$, evaluated from MC simulations.

\subsection{Fit model and results}

To extract the $\mathrm{B}_{c}^{+}$lifetime a binned chi-square fit to the ratio of the efficiency-corrected $c t$ distribution of the $\mathrm{B}_{c}^{+} \rightarrow \mathrm{J} / \psi \pi^{+}$and $\mathrm{B}^{+} \rightarrow \mathrm{J} / \psi \mathrm{K}^{+}$channels is used. The $\mathrm{B}_{c}^{+}$and $\mathrm{B}^{+}$signal $c t$ distributions from data are obtained by dividing the data sample in different $c t$ bins. In each bin an unbinned maximum-likelihood fit to the $\mathrm{J} / \psi \pi^{+}$and $\mathrm{J} / \psi \mathrm{K}^{+}$invariant mass distribution is performed, where the peak position and resolution is fixed to the values obtained from fits to the full samples, which are 

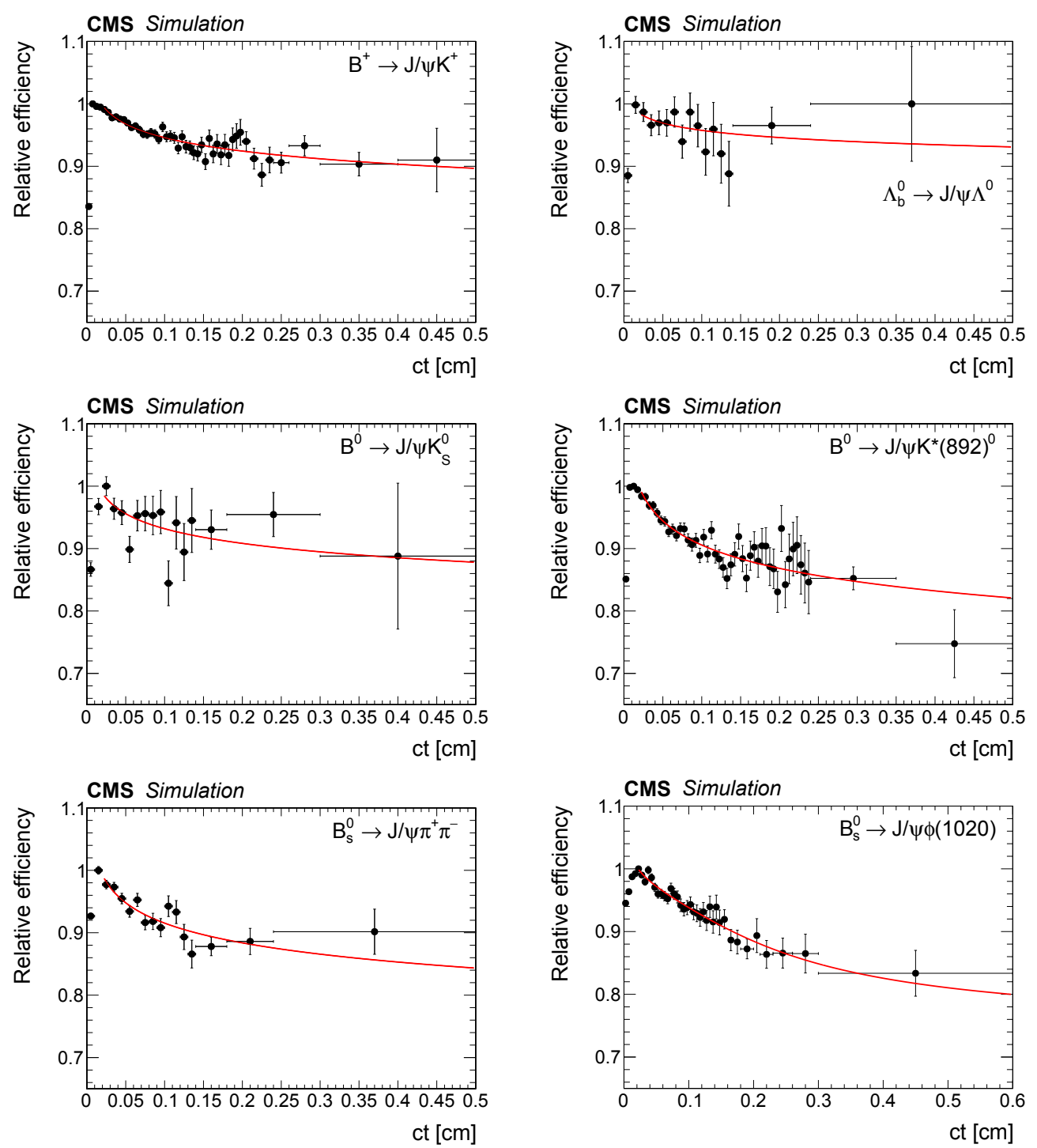

Figure 4. The reconstruction and selection efficiencies from simulation with a superimposed fit to an inverse power function as function of $c t$ for the different decay channels, $\mathrm{B}^{+} \rightarrow \mathrm{J} / \psi \mathrm{K}^{+}$(top left), $\mathrm{B}^{0} \rightarrow \mathrm{J} / \psi \mathrm{K}^{*}(892)^{0}$ (top right), $\mathrm{B}^{0} \rightarrow \mathrm{J} / \psi \mathrm{K}_{S}^{0}$ (center left), $\Lambda_{b}^{0} \rightarrow \mathrm{J} / \psi \Lambda^{0}$ (center right), $\mathrm{B}_{s}^{0} \rightarrow \mathrm{J} / \psi \pi^{+} \pi^{-}$(bottom left), and $\mathrm{B}_{s}^{0} \rightarrow \mathrm{J} / \psi \phi(1020)$ (bottom right) [2].

shown in Fig. 5. The bin edges, which are equal for the $\mathrm{B}_{c}^{+}$and $\mathrm{B}^{+} c t$ distribution are chosen to ensure similar statistical uncertainties in the $\mathrm{B}_{c}^{+}$signal yields among all bins. The $\mathrm{B}_{c}^{+}$and $\mathrm{B}^{+}$yields are shown in the left plot of Fig. 6 and the ratio of the two efficiency functions are shown in the right plot of Fig. 6. Figure 7 shows the ratio of the efficiency-corrected $c t$ distributions along with the result of a fit to an exponential function. The fit results in $\Delta \Gamma=4.12 \pm 0.30 \mathrm{c} / \mathrm{mm}$. 

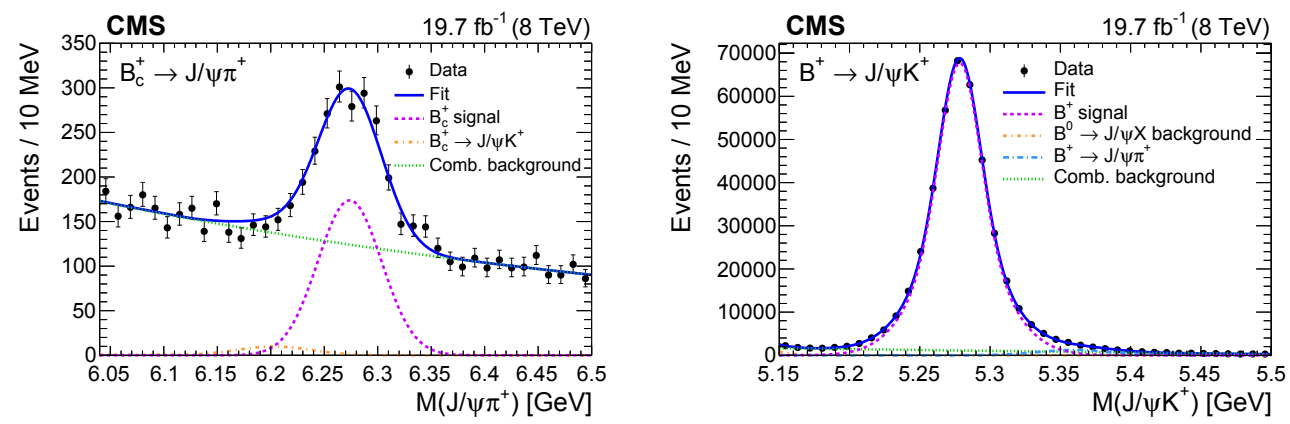

Figure 5. The $\mathrm{J} / \psi \pi^{+}$(left) and $\mathrm{J} / \psi \mathrm{K}^{+}$(right) invariant mass distribution. The solid line represents the total fit, the dashed line the signal component, and the dotted line the combinatorial background. Contributions from $\mathrm{B}_{c}^{+} \rightarrow \mathrm{J} / \psi \mathrm{K}^{+}, \mathrm{B}^{+} \rightarrow \mathrm{J} / \psi \pi^{+}$and $\mathrm{B}^{0} \rightarrow \mathrm{J} / \psi \mathrm{X}$ are also shown [2].
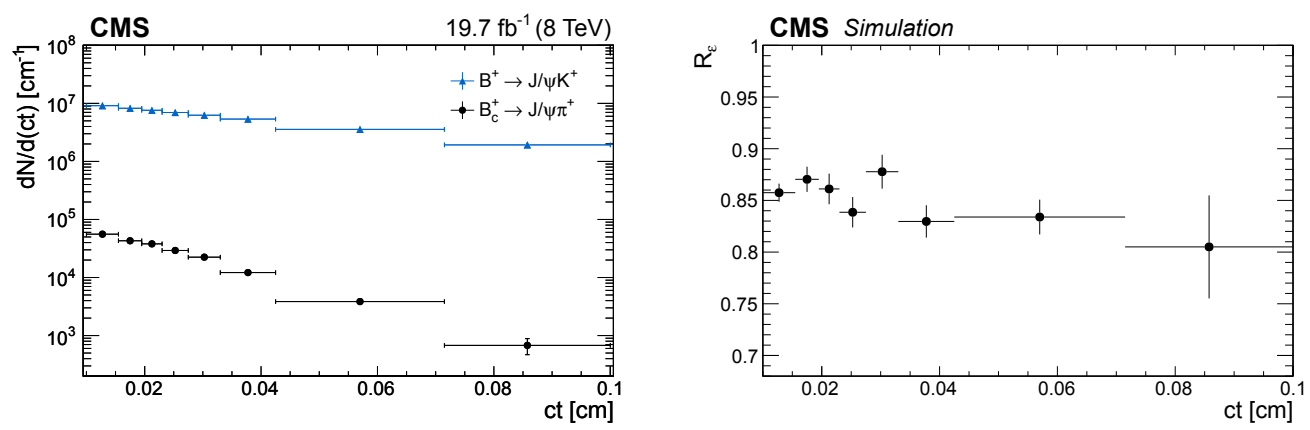

Figure 6. $\mathrm{B}_{c}^{+}$and $\mathrm{B}^{+}$signal yields normalized to the bin width (left) and ratio (right) of the $\mathrm{B}_{c}^{+}$and $\mathrm{B}^{+}$efficiency distributions as function of $c t$ [2].

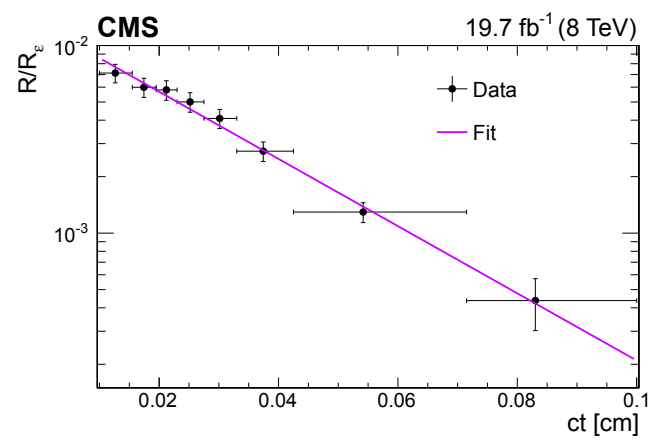

Figure 7. Ratio of the efficiency corrected $\mathrm{B}_{c}^{+}$to $\mathrm{B}^{+} c t$ distributions together with the result of the fit to an exponential function [2]. 


\section{Results}

The resulting lifetimes of the $\mathrm{B}^{0}, \mathrm{~B}_{s}^{0}$ and $\Lambda_{b}^{0}$ hadrons measured in the different decay channels are:

$$
\begin{array}{lr}
c \tau_{\mathrm{B}^{0}}=453.0 \pm 1.6 \text { (stat) } \pm 1.5 \text { (syst) } \mu \mathrm{m} & \text { in } \mathrm{J} / \psi \mathrm{K}^{*}(892)^{0}, \\
c \tau_{\mathrm{B}^{0}}=457.8 \pm 2.7 \text { (stat) } \pm 2.7 \text { (syst) } \mu \mathrm{m} & \text { in } \mathrm{J} / \psi \mathrm{K}_{S}^{0}, \\
c \tau_{\mathrm{B}_{s}^{0}}=504.3 \pm 10.5 \text { (stat) } \pm 3.7 \text { (syst) } \mu \mathrm{m} & \text { in } \mathrm{J} / \psi \pi^{+} \pi^{-}, \\
c \tau_{\mathrm{B}_{s}^{0}}=443.9 \pm 2.0 \text { (stat) } \pm 1.2 \text { (syst) } \mu \mathrm{m} & \text { in } \mathrm{J} / \psi \phi(1020), \\
c \tau_{\Lambda_{b}^{0}}=443.1 \pm 8.2 \text { (stat) } \pm 2.7 \text { (syst) } \mu \mathrm{m} & \text { in } \mathrm{J} / \psi \Lambda^{0} .
\end{array}
$$

Both measurements of the $\mathrm{B}^{0}$ lifetime are with the current world-average values, which are

$$
\begin{aligned}
c \tau_{\mathrm{B}^{0}} & =455.7 \pm 1.2 \mu \mathrm{m}[3], \\
c \tau_{\mathrm{B}_{s}^{0} \rightarrow \mathrm{J} / \psi \phi(1020)} & =443.4 \pm 3.6 \mu \mathrm{m}[16], \\
c \tau_{\mathrm{B}_{s}^{0} \rightarrow \mathrm{J} / \psi \pi^{+} \pi^{-}} & =497.1 \pm 9.6 \mu \mathrm{m} \mathrm{[16]} \\
c \tau_{\Lambda_{b}^{0}} & =440.7 \pm 3.0 \mu \mathrm{m} \mathrm{[3].}
\end{aligned}
$$

Using the result for $\Delta \Gamma$ from Section 5.1 and the lifetime of the $\mathrm{B}^{+}$meson, $c \tau_{\mathrm{B}^{+}}=491.1 \pm 1.2$ $\mu \mathrm{m}[3]$, the $\mathrm{B}_{c}^{+}$lifetime is found to be

$$
c \tau_{\mathrm{B}_{c}^{+}}=162.3 \pm 8.2(\text { stat }) \pm 4.7(\text { syst }) \pm 0.1\left(\tau_{\mathrm{B}^{+}}\right) \mu \mathrm{m},
$$

where the systematic uncertainty from the $\mathrm{B}^{+}$lifetime [3] is quoted separately. These results are in agreement with the average value from $\operatorname{LHCb}(153.4 \pm 2.8 \mu \mathrm{m})[11,12]$ and confirm a $\mathrm{B}_{c}^{+}$lifetime value higher than the average value measured at the Tevatron $(135.5 \pm 9.6 \mu \mathrm{m})[13-15]$.

\section{Summary}

The lifetimes of the $\mathrm{B}^{0}, \mathrm{~B}_{s}^{0}, \mathrm{~B}_{c}^{+}$and $\Lambda_{b}^{0}$ hadrons have been measured using different decay channels containing a $\mathrm{J} / \psi$ meson. The data, corresponding to an integrated luminosity of $19.7 \mathrm{fb}^{-1}$, have been collected by the CMS experiment in proton-proton collisions at a center-of-mass energy of $8 \mathrm{TeV}$. All measured lifetimes are compatible with the current world-average values. The precision of each channel is comparable or better than previous measurements in the respective channel for the neutral hadrons. The $\mathrm{B}_{c}^{+}$lifetime is in agreement with results from $\mathrm{LHCb}$ and confirms a longer lifetime than previously reported by the Tevatron experiments.

\section{References}

[1] A. Lenz, Int. J. Mod. Phys. A30, 1543005 (2015), arXiv: 1405. 3601

[2] A.M. Sirunyan et al. (CMS collaboration) (2017), CMS-BPH-13-008, CERN-EP-2017-244. Subm. to Eur. Phys. J. C., arXiv: 1710.08949

[3] C. Patrignani, Particle Data Group, Chinese Physics C 40, 100001 (2016)

[4] The CMS collaboration, Journal of Instrumentation 7, P10002 (2012), arXiv: 1206.4071

[5] V. Khachatryan et al. (CMS collaboration), Eur. Phys. J. C70, 1165 (2010), arXiv: 1007.1988

[6] V. Khachatryan et al. (CMS collaboration), Phys. Lett. B757, 97 (2016), arXiv: 1507.07527 
[7] R. Aaij et al. (LHCb), Phys. Rev. D87, 052001 (2013), arXiv: 1301.5347

[8] R. Aaij et al. (LHCb), Phys. Rev. D89, 092006 (2014), arXiv: 1402.6248

[9] A.S. Dighe, I. Dunietz, R. Fleischer, Eur. Phys. J. C6, 647 (1999), arXiv: hep-ph/9804253

[10] V.V. Kiselev (2002), arXiv:hep-ph/0211021

[11] R. Aaij et al. (LHCb collaboration), Phys. Lett. B742, 29 (2015), arXiv: 1411.6899

[12] R. Aaij et al. (LHCb collaboration), Eur. Phys. J. C74, 2839 (2014), arXiv: 1401.6932

[13] A. Abulencia et al. (CDF Collaboration), Phys. Rev. Lett. 97, 012002 (2006)

[14] T. Aaltonen et al. (CDF Collaboration), Phys. Rev. D 87, 011101 (2013)

[15] V.M. Abazov et al. (D0 Collaboration), Phys. Rev. Lett. 102, 092001 (2009)

[16] Heavy Flavor Averaging Group, ArXiv e-prints (2016), arXiv: 1612.07233 [hep-ex] 